\title{
Resultados preliminares de las competencias escritas (dimensión sintáctica) identificadas en textos elaborados por estudiantes de Educación
}

\author{
Preliminary results of the written competencies \\ (syntactic dimension) identified in passages written \\ by education student-teachers
}

Gisela Quintero de Chacón Universidad Nacional de Educación (Ecuador) gishelinaq@hotmail.com

Ana Delia Barrera Jiménez Universidad Nacional de Educación (Ecuador)

Teresa de Jesús Molina Gutiérrez Universidad Pedagógica Experimental Libertador

(Venezuela)

Revista Cumbres Vol.3 №2

Versión impresa ISSN 1390-9541

Versión electrónica ISSN 1390-3365

http://investigacion.utmachala.edu.ec/revistas/index.php/Cumbres 


\section{RESUMEN}

La escritura académica en las universidades es un indicador fundamental de desarrollo, requiere la adquisición y aplicación de competencias que satisfagan las demandas sociales y profesionales que le son inherentes. Específicamente, en la formación de profesionales de la Educación adquiere una connotación especial la práctica de competencias para la escritura como garantía para producir nuevos significados y para el tratamiento didáctico. A pesar de que se han hecho esfuerzos por mejorar la escritura académica persisten las dificultades en la producción escrita de los estudiantes universitarios, por ello el objetivo general de la investigación es: describir la dimensión sintáctica en los textos elaborados por los estudiantes de la carrera de Educación de la Universidad Pedagógica Experimental Libertador, Mérida-Venezuela. Se realizó un estudio mixto que dio preeminencia al paradigma cualitativo y empleó las técnicas del Análisis del discurso. La investigación se llevó a cabo en cuatro fases: I) estudio del estado del arte, II) diseño y construcción de los instrumentos de recogida de datos, III) proceso de validación de los instrumentos y IV) análisis de los resultados. Las debilidades encontradas en la muestra analizada demuestran un uso muy precario de competencias lingüísticas básicas (dimensión sintáctica), que en el nivel universitario deberían estar consolidadas. Estos resultados permitirán concebir acciones concretas desde el punto de vista didáctico, que actúen en favor de propuestas innovadoras para el mejoramiento del proceso de escritura en la formación de profesionales de la Educación.

Palabras clave: Estudiantes de educación, escritura académica, dimensión sintáctica.

\section{ABSTRACT}

Academic writing in universities is a fundamental indicator of development. It requires the acquisition and application of competencies that meet the social and professional demands that are inherent to it. The practice of writing skills acquires a special connotation of guarantee for new meanings and for didactic treatment in the training of education professionals. Although efforts have been made to improve academic writing, there are still difficulties in the written production of university students. The general objective of this study is to describe the syntactic dimension in texts written by students of the Education Career at Universidad Pedagógica Experimental Libertador, Mérida-Venezuela. A mixed study was conducted to give preeminence to the qualitative paradigm. The authors also used the techniques of Discourse Analysis. The research was performed in four phases: I) study of the state of the art, II) design and construction of data recognition instruments, III) validation process of the instruments and IV) analysis of results. The weaknesses found in the samples analyzed demonstrate a very precarious use of basic linguistic skills (syntactical dimension), which at the university level should already be consolidated. These results allow us to devise concrete 
actions from the didactic point of view, so that we can act in favor of innovative proposals for the improvement of the writing process in the training of Education professionals.

Keywords: Education student-teachers, academic writing, syntactic dimension.

\section{INTRODUCCIÓN}

La escritura académica en las universidades es un indicador esencial de desarrollo, requiere la adquisición y aplicación de competencias que satisfagan las demandas sociales y profesionales que le son inherentes. Específicamente, en la formación de profesionales de la Educación adquiere una connotación especial la práctica de competencias para la escritura como garantía para contribuir con una formación de excelencia.

El estudio exploratorio realizado acerca de los aportes con relación a este tema permitió escoger como teorías de apoyo algunos principios de la alfabetización académica (revisiones y actualización, Carlino, 2013), enfoques acerca de la lingüística del texto y su enseñanza (Ferrer y Rodríguez, 2010) y consideraciones relativos a los procesos de lectura y escritura (Parodi, 2010). Perspectivas y posturas que facilitaron constatar que si bien se aprecian avances desde el punto de vista didáctico, en la organización del proceso de enseñanza-aprendizaje de la escritura, no se logran erradicar las insuficiencias que caracterizan al proceso y al producto por parte de los estudiantes, a tal punto que se habla de un analfabetismo académico (Carlino, 2013); lo que revela un antagonismo entre lo que se desea y la realidad que continúa evidenciándose con relación a la comunicación escrita. Contradicción que se agudiza en la formación de profesionales de la Educación, quienes a su vez tienen a su cargo el tratamiento didáctico de dicho proceso en sus futuros alumnos.

La escritura, por ende, se convierte en un punto de mira de extraordinaria importancia, que va más allá del profesor de Lengua de las instituciones universitarias en donde se imparte la carrera de Educación, para convertirse en un problema de todos, con sobrada razón, se afirma que todo docente es un profesor de lengua, si tenemos en cuenta el papel que esta desempeña en los procesos de transmisión y adquisición de conocimientos en todas las asignaturas, lo que nos permite definirla como "eje transversal del currículum" (León. Álvarez y Tamayo, 2015).

Las aulas universitarias no enseñan a escribir, mucho menos a escribir los géneros más utilizados en el contexto académico, sin embargo, esas competencias son requeridas como parte de su actividad de estudio. Los estudiantes llegan al nivel universitario repitiendo información, copiándola y cuando logran escribir es a partir de un gran esfuerzo que no siempre conduce a los mejores resultados; por lo tanto, escribir es una competencia para la que no se ha preparado a los estudiantes (García y Villegas, 2015); como bien sostiene Carlino (2004) la escritura permanece como una práctica om- 
nipresente pero inobservable, práctica que se exige pero que no suele ser objeto de enseñanza.

Es indudable que el problema tiene sus causas en las insuficiencias de la didáctica del texto escrito. Se continúa enseñando desde enfoques tradicionales en los diferentes niveles del sistema educativo, lo que significa que predomina la influencia del modelo normativo de la enseñanza de la Lengua, Mostacero (2012) la llama pedagogía del error por su excesivo énfasis en los aspectos superficiales del texto, como son la ortografía, la acentuación, la puntuación o los recursos de cohesión gramatical, por lo que se deja de lado la variedad textual con sus propias estructuras y condiciones pragmáticas de producción. Hay que recordar que todavía lo más importante es el producto y no el proceso, basta sólo con observar cómo, quién y qué se corrige en un texto. No es sino a partir de las investigaciones de la Lingüística del texto que se ha dado una importancia preponderantemente comunicativa a la escritura académica, basada en el análisis textual, lo que ha generado cambios en su concepción y, por ende, en su enseñanza (Carlino 2012.

Escribir es un proceso complejo que comprende varias habilidades, requiere de su enseñanza planificada e incorporada al currículo, no sólo como una asignatura del Plan de Estudios de la carrera, sino que debe atravesar transversalmente todo el Pensum como un eje, idea que ya ha sido planteada por Carlino (2012); pero que actualmente se acompaña de un nuevo enfoque para escribir, y es desde las disciplinas (Carlino, 2014); lo que debería conducir a un programa integral direccionado por las instituciones. Llevar a cabo estas acciones no ha sido sencillo, de allí que desde hace unas décadas en Universidades australianas y estadounidenses se han creado los llamados Centros o Programas de escritura académica como una vía para contribuir con la solución de las insuficiencias escriturales de los estudiantes universitarios.

Los problemas que se presentan con la producción escrita de los estudiantes no son particulares de un país, ni de una universidad, es un asunto que debe involucrar a todos los países y a todos los profesionales de la docencia; controversia que se agudiza aún más en los estudiantes de Educación, puesto que son ellos los que van a enseñar a sus estudiantes a escribir y a asumir la escritura con función epistémica o no.

En las últimas décadas se han realizado investigaciones y estudios que nos sirven de referencia para contextualizar y obtener datos recientes acerca de las deficiencia en la producción escrita de textos académicos y no académicos (Pimienta, 2012, Carlino, 2013). Por su parte, Mostacero, (2014) y Molina y López, (2015) dan cuenta de la preocupación existente acerca de las deficiencias detectadas en la enseñanza de la lengua en todos los niveles de la escolaridad, así como destacan la incapacidad de los estudiantes de maestría para ajustar sus tesis de grado al género discursivo correspondiente.

Teniendo en cuenta las carencias evidenciadas y la necesidad de conjugar la enseñanza y el aprendizaje de la lectura y la escritura académica para encontrar alternativas de solución a la problemática descrita, la cual no solo afecta al ámbito académico sino también a la población general, nos planteamos el siguiente propósito investigativo: 


\section{Objetivo General}

Describir la dimensión sintáctica en los textos elaborados por los estudiantes de la carrera de Educación de la Universidad Pedagógica Experimental Libertador, Mérida-Venezuela.

\section{MATERIALES Y MÉTODOS}

En la investigación se asumió un diseño mixto que integró técnicas para valorar cualidades y ponderar rasgos, entonces se empleó la complementariedad metodológica o el diseño integral Hernández y Fernández, (2010) como proceso complejo que implica el estudio del fenómeno en todas sus manifestaciones y relaciones. No obstante, se dio preeminencia al paradigma cualitativo al tomar en cuenta la naturaleza del objeto de investigación: producción escrita (rasgos sintácticos) de los estudiantes. De manera que se describieron las características del fenómeno, obteniendo los datos directamente de la realidad (diseño de campo). Asimismo, se empleó el Análisis del Discurso (Van Dijk, 2002) para hacer una caracterización de los rasgos sintácticos que estructuran los textos escritos elaborados por los estudiantes de la carrera de Educación.

Con este fin se aplicó una prueba diagnóstica, la misma se organizó en tres partes. 1: datos generales. 2: lectura de texto para responder preguntas de comprensión lectora, 3.1: elaboración de un texto argumentativo (tres párrafos) a partir de un tópico dado y 3.2: redacción de un texto (tres párrafos) considerando una imagen presentada.

Para la descripción de los textos, lo que permitió valorar sus características, se empleó una escala que da cuenta de 5 niveles de evaluación (Excelente, Muy bien, Bien, Regular y Mal), para cada indicador considerado; de los cuales se interpretó el que obtuvo el mayor porcentaje de frecuencia de aparición. Mediante esa escala se evaluó, para este avance, sólo la dimensión sintáctica de los textos (integrada por 7 rasgos).

La técnica que se usó para la interpretación de los datos fue el análisis de contenido, la cual facilitó abordar los procesos los textos para describir sus estructuras sintácticas en el nivel textual.

En este avance parcial de la investigación se presentan sólo los rasgos descritos para la dimensión sintáctica, identificados en 41 textos, elaborados por 41 estudiantes de la carrera de Educación, en las menciones de: Inicial, Integral y Especial (Ubicados en $1^{\circ}, 2^{\circ}$ y $3^{\circ}$ semestre) de la Universidad Pedagógica Experimental Libertador en Mérida, Venezuela.

\section{RESULTADOS Y DISCUSIÓN}

\section{Análisis de la Dimensión Sintáctica}

Los aspectos considerados en la dimensión sintáctica son los que otorgan la cohesión textual, éstos facilitan la relación de cada enunciado con los demás 
a través de procedimientos lógico-sintácticos, léxico-semánticos y gramaticales, así como funcionan como señales que inciden en la adecuada interpretación del texto (Bentivoglio y Sedano, 2011).

En consecuencia, el empleo de las pautas gramaticales involucra gran importancia como instrumento de regulación de la actividad de escritura, por lo cual los escritores competentes han tenido que incorporar, como una habilidad imprescindible, la corrección gramatical en atención a las reglas del sistema lingüístico y a la norma gramatical

Tabla 1. Uso de los signos de puntuación según la intención comunicativa: coma, punto, punto y seguido, punto y aparte y dos puntos

\begin{tabular}{|c|c|c|c|c|c|c|c|c|c|c|c|c|}
\hline \multirow[t]{3}{*}{$\begin{array}{l}\text { Dimensión: } \\
\text { Sintáctica }\end{array}$} & \multicolumn{2}{|c|}{$\begin{array}{c}\text { Excelente } \\
5\end{array}$} & \multicolumn{2}{|c|}{$\begin{array}{c}\text { Muy } \\
\text { Bien } \\
4\end{array}$} & \multicolumn{2}{|c|}{$\begin{array}{c}\text { Bien } \\
3\end{array}$} & \multicolumn{2}{|c|}{$\begin{array}{c}\text { Regular } \\
2\end{array}$} & \multicolumn{2}{|c|}{$\begin{array}{c}\text { Mal } \\
1\end{array}$} & \multirow[t]{2}{*}{$\begin{array}{c}\text { TOTA } \\
\text { L }\end{array}$} & \multirow[t]{2}{*}{$\%$} \\
\hline & $\mathrm{F}$ & $\%$ & $\mathrm{~F}$ & $\%$ & $\mathrm{~F}$ & $\%$ & $\mathrm{~F}$ & $\%$ & $\mathrm{~F}$ & $\%$ & & \\
\hline & 0 & 0 & 3 & 7,3 & 9 & 21,9 & 24 & 58,5 & 5 & 12,1 & 41 & 99,8 \\
\hline
\end{tabular}

Nota. Datos tomados del instrumento aplicado a los estudiantes, (2016)

Acerca del empleo del indicador Uso de los signos de puntuación según la intención comunicativa: coma, punto y seguido, punto y aparte y dos puntos, es preciso señalar que el 58,5 de los evaluados se ubicó en la calificación Regular, sin duda que al emplear de modo regular los signos de puntuación están restándole significado, funcionalidad y sentido al texto.

En el ejemplo 1 se puede observar la ausencia total de los signos de puntuación, sólo se usa el punto y aparte.

Ejemplo 1

El aborto

En este siglo la situación de aborto a [ha] sido de gran impacto para Venezuela ya que se encuentran adolescentes insegura de si [sí] mismas y tienden a salir embarazada y no tienen la más minina idea del compromiso que eso trae por lo tanto toman la decisión de abortar causando daño a un ser humano y un daño psicológicamente.

Tabla 2. Uso y pertinencia de los conectores

\begin{tabular}{|l|l|l|l|l|l|l|l|l|l|l|l|l|}
\hline Dimensión: & \multicolumn{2}{|c|}{$\begin{array}{c}\text { Excelente } \\
\text { Sintáctica }\end{array}$} & \multicolumn{2}{|c|}{$\begin{array}{c}\text { Muy } \\
\text { Bien } \\
4\end{array}$} & \multicolumn{2}{|c|}{$\begin{array}{c}\text { Bien } \\
3\end{array}$} & \multicolumn{2}{|c|}{$\begin{array}{c}\text { Regular } \\
2\end{array}$} & \multicolumn{2}{|c|}{$\begin{array}{c}\text { Mal } \\
1\end{array}$} & $\begin{array}{c}\text { TOTA } \\
\text { L }\end{array}$ & $\%$ \\
\cline { 2 - 14 } & F & $\%$ & F & $\%$ & F & $\%$ & F & $\%$ & F & $\%$ & & \\
\cline { 2 - 15 } & 1 & 2,4 & 1 & 2,4 & 8 & 19,5 & 16 & 39 & 15 & 36,5 & 41 & 99,8 \\
\hline
\end{tabular}

Nota. Datos tomados del instrumento aplicado a los estudiantes, (2016)

Con relación al indicador Uso y pertinencia de los conectores, el 39\% de los estudiantes se encuentran en la calificación Regular. Al respecto es importante comentar que en el corpus predomina un uso muy elemental de los conectores, por ello existe una importante dificultad para indicar mediante los conectores correctos las intenciones que el escritor quiere explicitar. En la mayoría de los textos argumentativos elaborados por los estudiantes 
son escasos los conectores de oposición, de concesión o de conclusión, estos son esenciales para indicar la orientación argumentativa. En el ejemplo 2 se puede constatar la ausencia de conectores tanto en el interior de los párrafos, como para establecer las conexiones entre los párrafos que integran el texto

Ejemplo 2

Considero una irresponsabilidad por parte del hombre y la mujer al tener relaciones sexuales sin ninguna protección teniendo como consecuencia embarazos no deseados El aborto es un crimen que no se debe permitir pues son niños inocentes que no tienen la culpa de que sus padres sean unos irresponsables

El aborto debe ser más penalizado porque son cosas que no se deben permitir tomando en cuenta que nosotros como mujeres debemos ser responsables con nuestros actos.

Tabla 3. Uso de adverbios

\begin{tabular}{|c|c|c|c|c|c|c|c|c|c|c|c|c|}
\hline \multirow[t]{3}{*}{$\begin{array}{l}\text { Dimensión: } \\
\text { Sintáctica }\end{array}$} & \multicolumn{2}{|c|}{$\begin{array}{c}\text { Excelente } \\
5\end{array}$} & \multicolumn{2}{|c|}{$\begin{array}{l}\text { Muy } \\
\text { Bien } \\
4\end{array}$} & \multicolumn{2}{|c|}{$\begin{array}{c}\text { Bien } \\
3\end{array}$} & \multicolumn{2}{|c|}{$\begin{array}{l}\text { Regular } \\
2\end{array}$} & \multicolumn{2}{|c|}{$\begin{array}{c}\text { Mal } \\
1\end{array}$} & \multirow[t]{2}{*}{$\begin{array}{c}\text { TOTA } \\
\text { L }\end{array}$} & \multirow[t]{2}{*}{$\%$} \\
\hline & F & $\%$ & $\mathrm{~F}$ & $\%$ & F & $\%$ & F & $\%$ & $\mathrm{~F}$ & $\%$ & & \\
\hline & 0 & 0 & 2 & 4,8 & 7 & 17 & 27 & 65,8 & 5 & 12,1 & 41 & 99,7 \\
\hline
\end{tabular}

Nota. Datos tomados del instrumento aplicado a los estudiantes, (2016)

Por otra parte, el indicador Uso de adverbios, reportó el 65,8\% de uso para la escala Regular. El empleo de los adverbios es un mecanismo gramatical de cohesión relacionado con la deixis temporal, son invariables y modificadores.

Se puede constatar en el corpus el uso inapropiado del adverbio de lugar donde, para indicar una idea relacionada con tiempo.

Ejemplo 3

(...) es un tema que se da mucho en los adolecentes [adolescentes] ya que es donde se provoca el aborto por la falta de comunicación (...)

Tabla 4. Uso del verbo haber

\begin{tabular}{|c|c|c|c|c|c|c|c|c|c|c|c|c|}
\hline \multirow[t]{3}{*}{$\begin{array}{l}\text { Dimensión: } \\
\text { Sintáctica }\end{array}$} & \multicolumn{2}{|c|}{$\begin{array}{c}\text { Excelente } \\
5\end{array}$} & \multicolumn{2}{|c|}{$\begin{array}{c}\text { Muy } \\
\text { Bien } \\
4\end{array}$} & \multicolumn{2}{|c|}{$\begin{array}{c}\text { Bien } \\
3\end{array}$} & \multicolumn{2}{|c|}{$\begin{array}{c}\text { Regular } \\
2\end{array}$} & \multicolumn{2}{|c|}{$\begin{array}{c}\text { Mal } \\
1\end{array}$} & \multirow[t]{2}{*}{$\begin{array}{c}\text { TOTA } \\
\text { L }\end{array}$} & \multirow[t]{2}{*}{$\%$} \\
\hline & $\mathrm{F}$ & $\%$ & $\mathrm{~F}$ & $\%$ & $\mathrm{~F}$ & $\%$ & $\mathrm{~F}$ & $\%$ & F & $\%$ & & \\
\hline & 1 & 2,4 & 5 & 12,1 & 5 & 12,1 & 26 & 63,4 & 4 & 9,7 & 41 & 99,7 \\
\hline
\end{tabular}

Nota. Datos tomados del instrumento aplicado a los estudiantes, (2016)

En lo que respecta al indicador Uso del verbo haber: había por habían, haya por hayan, hubo por hubieron, haya por haiga, somos por habemos, hace por hacen, los resultados indican que 63,4 de los evaluados se ubicó en la escala Regular.

El ejemplo 4 muestra una transgresión a la norma en el uso del verbo haber, muy común en contextos informales y, con menos frecuencia, en en- 
tornos formales: la pluralización del verbo haber. Se pluraliza inapropiadamente el verbo haber porque se cree que debe concordar en número con lo

Ejemplo 4

Casos que en la actualidad ya se nos han hecho común presenciar (...)

Tabla 5. Uso de términos unívocos

\begin{tabular}{|l|l|l|l|l|l|l|l|l|l|l|l|l|}
\hline $\begin{array}{l}\text { Dimensión: } \\
\text { Sintáctica }\end{array}$ & $\begin{array}{c}\text { Excelente } \\
5\end{array}$ & \multicolumn{2}{|c|}{$\begin{array}{c}\text { Muy } \\
\text { Bien } \\
4\end{array}$} & \multicolumn{2}{|c|}{$\begin{array}{c}\text { Bien } \\
3\end{array}$} & \multicolumn{2}{|c|}{$\begin{array}{c}\text { Regular } \\
2\end{array}$} & \multicolumn{2}{|c|}{$\begin{array}{c}\text { Mal } \\
1\end{array}$} & $\begin{array}{c}\text { TOTA } \\
\text { L }\end{array}$ & $\%$ \\
\cline { 2 - 15 } & F & $\%$ & F & $\%$ & F & $\%$ & F & $\%$ & F & $\%$ & & \\
\cline { 2 - 15 } & 0 & 0 & 3 & 7,3 & 5 & 12,1 & 12 & 29,2 & 21 & 51,2 & 41 & 99,8 \\
\hline
\end{tabular}

Nota. Datos tomados del instrumento aplicado a los estudiantes, (2016)

que supuestamente es el sujeto de la oración.

Con relación al rasgo Uso de términos unívocos, el $51,2 \%$ de los evaluados, se ubicó en la escala Mal ya que para emplear términos unívocos se requiere un manejo preciso del lenguaje técnico de la disciplina en cuestión, asimismo, es esencial mantener de modo constante la significación, sin que la misma esté sujeta a interpretaciones individuales.

En el ejemplo 5 se puede observar el empleo inapropiado del significado del término globalización, de igual manera predomina la ambigüedad con

Ejemplo 5

Sin embargo, la globalización es un factor que nos afecta a todos principalmente al ambiente ya que es este [éste] el que recibe la peor parte de todos estos cambios e innovación y confort que el hombre pretende alcanzar.

Tabla 6. Uso de la sinonimia

\begin{tabular}{|l|l|l|l|l|l|l|l|l|l|l|l|l|}
\hline Dimensión: & $\begin{array}{c}\text { Excelente } \\
\text { Sintáctica }\end{array}$ & \multicolumn{2}{|c|}{$\begin{array}{c}\text { Muy } \\
\text { Bien }\end{array}$} & \multicolumn{2}{|c|}{$\begin{array}{c}\text { Bien } \\
4\end{array}$} & \multicolumn{2}{|c|}{$\begin{array}{c}\text { Regular } \\
2\end{array}$} & \multicolumn{2}{|c|}{$\begin{array}{c}\text { Mal } \\
1\end{array}$} & $\begin{array}{c}\text { TOTA } \\
\text { L }\end{array}$ & $\%$ \\
\cline { 2 - 15 } & F & $\%$ & F & $\%$ & F & $\%$ & F & $\%$ & F & $\%$ & & \\
\cline { 2 - 14 } & 0 & 0 & 3 & 7,3 & 5 & 12,1 & 12 & 29,2 & 21 & 51,2 & 41 & 99,8 \\
\hline
\end{tabular}

Nota. Datos tomados del instrumento aplicado a los estudiantes, (2016)

relación al sentido que se le ha querido asignar.

Acerca del Uso de la sinonimia, el 65,8\% de los evaluados, se ubicó en la escala Regular, ello indica que existen regulares habilidades para sustituir un elemento léxico por otro, para establecer relaciones semánticas dentro del texto entre el término sustituido y el sustituto y por ende, para otorgar fluidez a la progresión temática (evita la repetición de palabras). La sinonimia corresponde a los mecanismos de cohesión léxica pues emplea los significados de los términos para establecer nexos entre las proposiciones, intervienen mecanismos de correferencialidad. 
En el ejemplo 6 se pueden evidenciar significativas dificultades para emplear el recurso de la sinonimia, por lo que el texto se hace repetitivo y no logra

Ejemplo 6

(...) Sin embargo algunas Organizaciones se oponen ya que algunas personas las utilizan para diversión y su uso causa en las personas falta de interés por realizar y ser responsables en sus actividades en la vida cotidiana (...)

Tabla 7. Uso de la elipsis con pronombres y con verbos

\begin{tabular}{|c|c|c|c|c|c|c|c|c|c|c|c|c|}
\hline \multirow[t]{3}{*}{$\begin{array}{l}\text { Dimensión: } \\
\text { Sintáctica }\end{array}$} & \multicolumn{2}{|c|}{$\begin{array}{c}\text { Excelente } \\
5\end{array}$} & \multicolumn{2}{|c|}{$\begin{array}{c}\text { Muy } \\
\text { Bien } \\
4\end{array}$} & \multicolumn{2}{|c|}{$\begin{array}{c}\text { Bien } \\
3\end{array}$} & \multicolumn{2}{|c|}{$\begin{array}{c}\text { Regular } \\
\quad 2\end{array}$} & \multicolumn{2}{|c|}{$\begin{array}{c}\text { Mal } \\
1\end{array}$} & \multirow[t]{2}{*}{$\begin{array}{c}\text { TOTA } \\
\text { L }\end{array}$} & \multirow[t]{2}{*}{$\%$} \\
\hline & $\mathrm{F}$ & $\%$ & F & $\%$ & F & $\%$ & $\mathrm{~F}$ & $\%$ & F & $\%$ & & \\
\hline & 0 & 0 & 4 & 9,7 & 7 & 17 & 16 & 39 & 14 & 34 & 41 & 99,8 \\
\hline
\end{tabular}

Nota. Datos tomados del instrumento aplicado a los estudiantes, (2016)

desarrollar el tema mediante proposiciones que lo armonicen como un todo.

En lo que respecta al uso de la elipsis con pronombres y con verbos, los resultados indican que el 39\% de los evaluados se ubica en la escala Regular, de lo cual se infiere que poseen destrezas calificables como regulares para hacer uso de la estrategia que permite la sustitución de un término en que el sustituto es cero, evita repeticiones y el receptor reconstruye la refe-

Ejemplo 7

(...) porque es algo que ayuda mucho a personas que tienen una enfermedad terminal $y$ lo que hacen [ellos] es sufrir, y sin embargo muchos (...)

rencia. Ejemplo 7:

\section{CONCLUSIONES}

Es necesario puntualizar que uno de los mecanismos esenciales en la tarea de escribir y sobre el cual se discutió en este estudio es la dimensión sintáctica (Uso de los signos de puntuación, Uso y pertinencia de los conectores, Uso de adverbios, Uso del verbo haber, Uso de términos unívocos, Uso de la sinonimia, Uso de adverbios, uso de la elipsis con pronombres y con verbos). La evaluación de estos mecanismos de cohesión se ubicó en la calificación de Regular y Mal, lo que significa que hay un uso precario de las normas que organizan la microestructura textual, entonces, en los textos analizados, las funciones de los vínculos cohesivos no se cumplieron, ni hubo continuidad lógico-semántica entre las proposiciones, oraciones y párrafos. Ello evidencia las dificultades que confrontan estos estudiantes para entender que, en su mayor parte, el texto se va construyendo a partir de lo ya dicho y que tiene características retrospectivas. 
Esa inadecuación a las normas sintácticas determinó que la mayoría de los textos estudiados se organizaran en frases aisladas, cargados de repeticiones innecesarias, abundantes transgresiones a las convenciones ortográficas, con predominancia del lenguaje coloquial, así como plagados de ambigüedades. Lo cual representa una gran limitación ya que el empleo adecuado de los recursos cohesivos repercute en la reducción del esfuerzo para procesar la información.

Asimismo, la preocupación que genera la precariedad comprobada en el manejo de los recursos sintácticos de estos estudiantes, es potenciada cuando se considera que la escritura académica, entendida como el registro lingüístico en el que se comunican los universitarios, es concebido como una forma de la lengua socialmente reconocida, mediante la cual se produce y legitima el conocimiento académico (Hernández, 2009); así que hacerse competente en su uso implica no sólo el éxito como estudiante, sino también contar con el poder para dirigirse y comunicarse con quienes "detentan las formas legítimas del lenguaje oral, escrito o multimodal” (Rojas, 2012, p.3), lo que además involucra el empleo del lenguaje con voluntad propia. En el mismo sentido, es impostergable que los estudiantes entiendan que las habilidades de leer y escribir son tan importantes para su desempeño académico como el hablar y escuchar lo es para sus actividades cotidianas.

Finalmente, es relevante precisar que, no obstante esta situación nada satisfactoria, es necesario tener muy en cuenta la complejidad que revisten los procesos de lectura y escritura, pues como lo indica Carlino (2004) comprensión, producción de textos y conocimiento son inseparables, asimismo no se puede obviar que estos estudiantes se están enfrentando a una cultura que les resulta ajena, tanto en las prácticas lingüísticas que necesitan manejar, como en el conocimiento de las áreas disciplinares pedagógicas que integran el currículo universitario.

\section{REFERENCIAS BIBLIOGRÁFICAS}

Bentivoglio, P. y Sedano, M. (2011). Problemas gramaticales en la escritura. En A. Bolívar y R. Beke. (Comp.), Lectura y escritura para la investigación (pp. 239-275). Caracas: Universidad Central de Venezuela.

Carlino, P. (2013). Alfabetización académica diez años después. Revista Mexicana de Investigación Educativa, 18(57), 355-381.

Carlino, P. (2012). Escribir a través del currículum: tres modelos para hacerlo en la universidad y una adenda. Cuadernos Pedagógicos, 1(1) 8-21.

Carlino, P. (2004). El proceso de escritura académica: Cuatro dificultades de la enseñanza universitaria. EDUCERE, 8(26), 321 -327.

Entrevista con Paula Carlino, Directora de GICEOLEM (2014). En S. Serrano y R. Mostacero (comp.) La escritura académica en Venezuela. Investigación, reflexión y propuestas (pp. 25-48). Mérida, Venezuela: Universidad de Los Andes.

Ferrer, M. y Rodríguez, C. (2010). La lingüística del texto en los manuales de 
la eso. En T. Ribas Seix (Coord.), Libros de texto y enseñanza de la gramática (pp. 97-116). Barcelona: Graó.

García, L. y Villegas, C. (2015). Lectura y escritura en la universidad: procesos fundamentales en la comunicación del conocimiento. REDHECS, 19(10), 121-137.

Hernández, S. y Fernández, B. (2010). Metodología de la investigación. La Habana: Félix Varela.

Hernández, G. (2009). Escritura académica y formación de maestros. ¿Por qué no acaban las tesis? Tiempo de Educar, 10(19), 11-40.

Molina, T. y López, A. (2015). El planteamiento del problema: un problema de competencias de escritura o de desconocimiento de la cultura discursiva de las disciplinas. Ponencia presentada en el I Jornada de Escritura y Lectura como Prácticas Académicas y Sociales. Universidad Pedagógica Experimental Libertador, Mérida.

Mostacero, R. (2014). La escritura en la investigación: un recorrido entre lo epistémico y lo pedagógico. En S. Serrano y R. Mostacero (Comp.), La escritura académica en Venezuela. Investigación, reflexión y propuestas (pp. 197205). Mérida, Venezuela: Universidad de Los Andes.

Mostacero, R. (2012). Dificultades de escritura en el discurso académico: Análisis crítico de una situación problemática. Legenda, 16(14), 64-88.

Parodi, G. (2010). Saber leer. Bogotá: Aguilar.

Pimienta, J. (2012). Estrategias de enseñanza aprendizaje. Docencia universitaria basada en competencias. México: Pearson.

León, M., Álvarez, Y., y Tamayo, M. (2015). El texto científico-profesional: necesidad de la Educación Superior. Universidad y Sociedad, 7(3), 187-195.

Rojas, E. (2012). Las investigaciones sobre escritura académica realizadas en la Universidad Simón Bolívar (USB): Un somero estado del arte. Lengua y Habla, 16(226), 1-16.

van Dijk, T. (2002). El análisis crítico del discurso y el pensamiento social. Athenea Digital. Num.1 primavera. 


\section{Cumbres}

